\title{
Local strain behavior of bulk metallic glasses under tension studied by in situ $x$-ray diffraction
}

\author{
X. D. Wang, ${ }^{1, a)}$ J. Bednarcik, ${ }^{2}$ H. Franz, ${ }^{2}$ H. B. Lou, ${ }^{1}$ Z. H. He, ${ }^{1}$ Q. P. Cao, ${ }^{1}$ and \\ J. Z. Jiang ${ }^{1, b)}$ \\ ${ }^{1}$ Department of Materials Science and Engineering, Laboratory of New-Structured Materials, \\ Zhejiang University, Hangzhou 310027, People's Republic of China and International Center \\ for New-Structured Materials (ICNSM), Zhejiang University, Hangzhou 310027, People's Republic of China \\ ${ }^{2}$ HASYLAB, DESY, Notkestrasse 85, D-22603 Hamburg, Germany
}

(Received 14 November 2008; accepted 12 December 2008; published online 7 January 2009)

\begin{abstract}
The uniaxial tensile behavior of $\mathrm{Zr}_{62} \mathrm{Al}_{8} \mathrm{Ni}_{13} \mathrm{Cu}_{17}, \quad \mathrm{Cu}_{46} \mathrm{Zr}_{46} \mathrm{Al}_{8}, \mathrm{Zr}_{48} \mathrm{Cu}_{43} \mathrm{Al}_{7} \mathrm{Ag}_{2}$, and $\mathrm{La}_{62} \mathrm{Al}_{14}\left(\mathrm{Cu}_{5 / 6} \mathrm{Ag}_{1 / 6}\right){ }_{14} \mathrm{Co}_{5} \mathrm{Ni}_{5}$ bulk metallic glasses (BMGs) has been investigated by using in situ synchrotron radiation diffraction technique. It is found that the local strain is basically homogeneously distributed at low stress. However, heterogeneity appears obviously when the stress is close to the fracture strength. The amplitude of fluctuation in local strain for four BMGs could relate to the distribution of excess free volume within the medium range order. (C) 2009 American Institute of Physics. [DOI: 10.1063/1.3064136]
\end{abstract}

Mechanical properties of bulk metallic glasses (BMGs) are currently of great interest. ${ }^{1-3}$ One key issue is the failure mechanism of BMGs upon loading, which still remains unclear. Under uniaxial compression or bending conditions, some BMGs exhibit pronounced plasticity. ${ }^{4,5}$ More recently, pronounced tensile ductility for BMG composites was also reported. ${ }^{6}$ However, no tensile plasticity has been found so far for monolithic BMGs at ambient temperature and by uniaxial tension. The shear transformation zone (STZ) and free volume (or open volume) model were proposed to interpret the shear band formation from the local structural change in BMGs. ${ }^{7,8}$ To further understand the evaluation of STZ or the distribution of free volume upon loading, the deformation at the stress close to the fracture strength is greatly concerned. Usually the elastic deformation of BMGs is regarded to be similar to that of most crystalline counterparts, linearly and reversibly. However, some reports show that preloading below the fracture strength of BMGs can enhance excess free volume and produce extended plasticity upon reloading, indicating that some irreversible effect happens in the elastic deformation. ${ }^{9}$ In this work, we report different local atomic responses to tensile stress for four BMGs investigated by in situ high energy $\mathrm{x}$-ray diffraction (HEXRD), which was also used to measure the elastic strain under compression ${ }^{10,11}$ and to detect the change in excess free volume upon heating ${ }^{12}$ and homogeneous plastic deformation ${ }^{13}$ of BMGs.

Using high purity raw materials, prealloyed ingots of nominal compositions $\mathrm{Zr}_{62} \mathrm{Al}_{8} \mathrm{Ni}_{13} \mathrm{Cu}_{17}, \mathrm{Cu}_{46} \mathrm{Zr}_{46} \mathrm{Al}_{8}$, $\mathrm{Zr}_{48} \mathrm{Cu}_{43} \mathrm{Al}_{7} \mathrm{Ag}_{2}$, and $\mathrm{La}_{62} \mathrm{Al}_{14}\left(\mathrm{Cu}_{5 / 6} \mathrm{Ag}_{1 / 6}\right)_{14} \mathrm{Co}_{5} \mathrm{Ni}_{5}$ were prepared by arc melting under a Ti-gettered purified argon atmosphere. From the ingots, sheet-shaped samples $1 \mathrm{~mm}$ thick, $10 \mathrm{~mm}$ wide, and $60 \mathrm{~mm}$ long were prepared by suction casting in a water-cooled copper mold. Ultrasonic measurements were used a MATEC 6600 model ultrasonic system under a frequency of $5 \mathrm{MHz}$. Moreover, densities were

\footnotetext{
${ }^{\text {a) }}$ Author to whom correspondence should be addressed. Electronic mail: wangxd@zju.edu.cn.

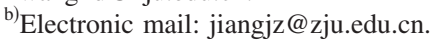

measured in a Mettler Toledo XS105 microbalance with a sensitivity of $0.01 \mathrm{mg}$. The experimental details for tension by using synchrotron radiation can be referred to Ref. 14 .

The increase in average interatomic distance can be reflected by the change of the position of the first maximum in $I(q)$ [or $S(q)$ ] or the peak positions in $G(r))^{11,12}$ Thus, the elastic strain under stress for BMGs can be evaluated by using $q_{0}$ and $q, \varepsilon=\left(q_{0}-q\right) / q$, where $\varepsilon$ is the elastic strain, and $q_{0}$ and $q$ are the first-peak positions in $I(q)$ [or $S(q)$ ] under zero stress and stress $\sigma$, respectively. The peak positions of the first peak in $I(q)$ were accurately fitted by using Voigt function in the software package of PEAKFIT. Figure 1 shows the tensile stress as a function of strain calculated by the above equation. The data of $I(q)$ integrated along the tensile direction record the contribution of all atomic pairs parallel to the tensile direction in the sample. Thus, the shift in the first peak position under tension should reflect the change in the average interatomic distance in samples upon tension, i.e., the magnitude of microscopic strain. By linearly fitting the relationship of stress with strain for each alloy in Fig. 1, the tensile elastic moduli are obtained to be about 83 GPa for $\mathrm{Zr}_{62} \mathrm{Al}_{8} \mathrm{Ni}_{13} \mathrm{Cu}_{17}, 91 \mathrm{GPa}$ for $\mathrm{Zr}_{48} \mathrm{Cu}_{43} \mathrm{Al}_{7} \mathrm{Ag}_{2}$, $102 \mathrm{GPa}$ for $\mathrm{Cu}_{46} \mathrm{Zr}_{46} \mathrm{Al}_{8}$, and $34 \mathrm{GPa}$ for

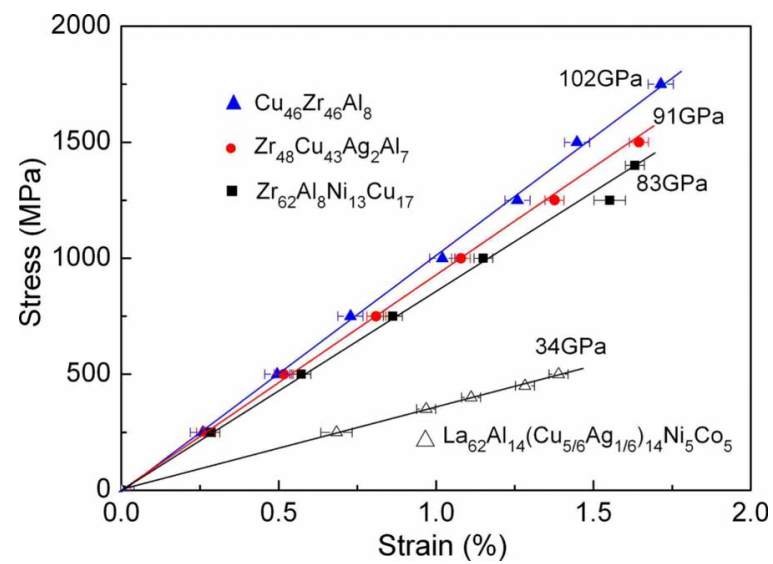

FIG. 1. (Color online) Tensile stress-strain curves of four BMGs obtained from the peak shift in the first maximum in $I(q)$ by in situ HEXRD. 


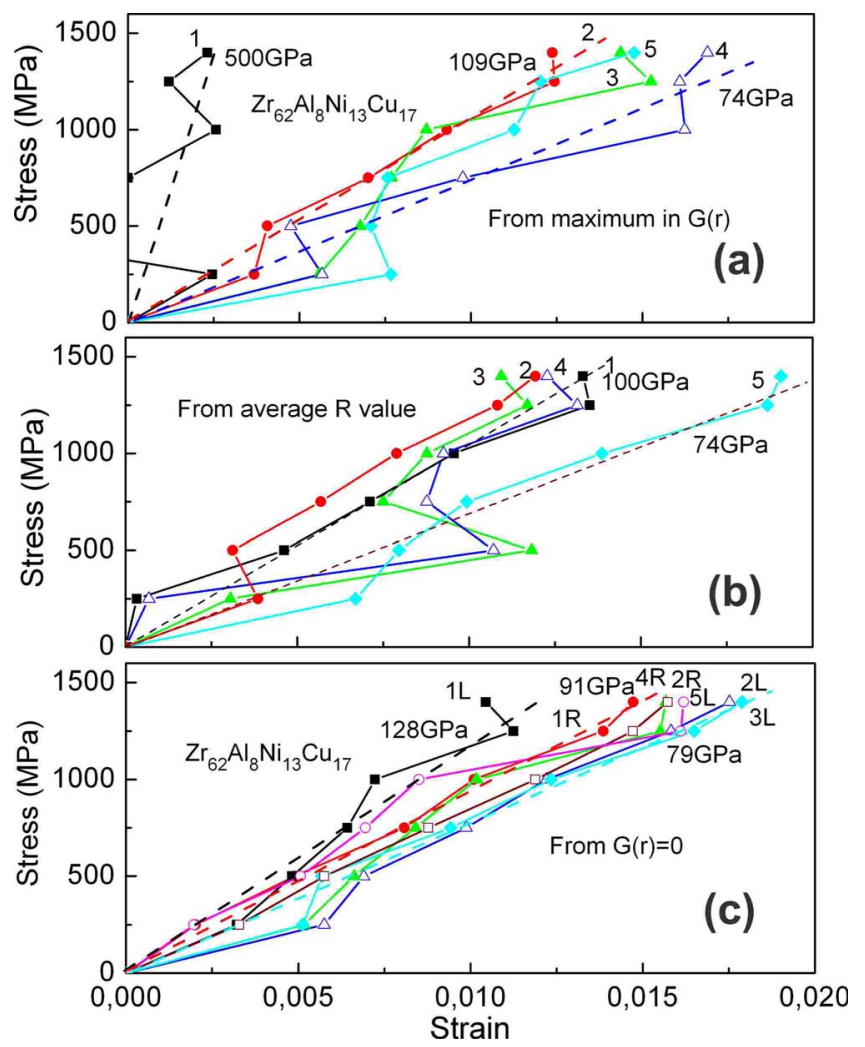

FIG. 2. (Color online) Tensile stress-strain curves for $\mathrm{Zr}_{62} \mathrm{Al}_{8} \mathrm{Ni}_{13} \mathrm{Cu}_{17} \mathrm{BMG}$ got by three different methods based on $G(r)$ : (a) from the shift of the local maximum, (b) from the average $r$ value of each shell, and (c) from the points with $G(r)=0$.

$\mathrm{La}_{62} \mathrm{Al}_{14}\left(\mathrm{Cu}_{5 / 6} \mathrm{Ag}_{1 / 6}\right)_{14} \mathrm{Co}_{5} \mathrm{Ni}_{5}$ BMGs. These results are in good agreement with the experimental values measured by ultrasonic method, $80 \mathrm{GPa}$ for $\mathrm{Zr}_{62} \mathrm{Al}_{8} \mathrm{Ni}_{13} \mathrm{Cu}_{17}, 90 \mathrm{GPa}$ for $\mathrm{Zr}_{48} \mathrm{Cu}_{43} \mathrm{Al}_{7} \mathrm{Ag}_{2}, 96 \mathrm{GPa}$ for $\mathrm{Cu}_{46} \mathrm{Zr}_{46} \mathrm{Al}_{8}$, and $35 \mathrm{GPa}$ for $\mathrm{La}_{62} \mathrm{Al}_{14}\left(\mathrm{Cu}_{5 / 6} \mathrm{Ag}_{1 / 6}\right)_{14} \mathrm{Co}_{5} \mathrm{Ni}_{5}$ BMGs. The agreement strongly indicates that in situ HEXRD using $I(q)$ data is an effective means to probe the tensile behavior of BMGs.

$G(r)$ data reveal the average probabilities for finding atoms at a distance $r$ for a given atom. Unlike discrete shell distances in crystalline phases, atoms in glasses are considered to be located in shells, which usually exhibit a broad distribution. Under tension, the peaks in $G(r)$ also shift. Thus, one question is whether one can deduce the information for local atomic responses to stress during tension from $G(r)$ data by using $r_{0}$ and $r$, as in $\varepsilon=\left(r-r_{0}\right) / r_{0}$, where $r_{0}$ and $r$ denote the positions of peak in $G(r)$ under zero stress and stress $\sigma$, respectively. To address this question, we further analyzed $G(r)$ curves as a function of stress. The pair distribution function $G(r)$ of each sample was obtained by Fourier transformation of the $S(q)$ data. The peak shape in $G(r)$ curves usually exhibits asymmetry and peak splitting. Therefore, it is of great importance to know which way is suitable for estimating the position shift of each shell. In the following, we compared three different ways that are used to estimate the local strain based on the peak shift in $G(r)$ curves for the $\mathrm{Zr}_{62} \mathrm{Al}_{8} \mathrm{Ni}_{13} \mathrm{Cu}_{17} \mathrm{BMG}$. One approach is based on the determination of the maximum in each peak. The positions of maxima at various shells in $G(r)$ under stress are yielded by using the package of PEAKFIT. Figure 2(a) shows the stress versus strain curves obtained from the first five maxima using Eq. (2). In this case, the stiffness is extraordi-

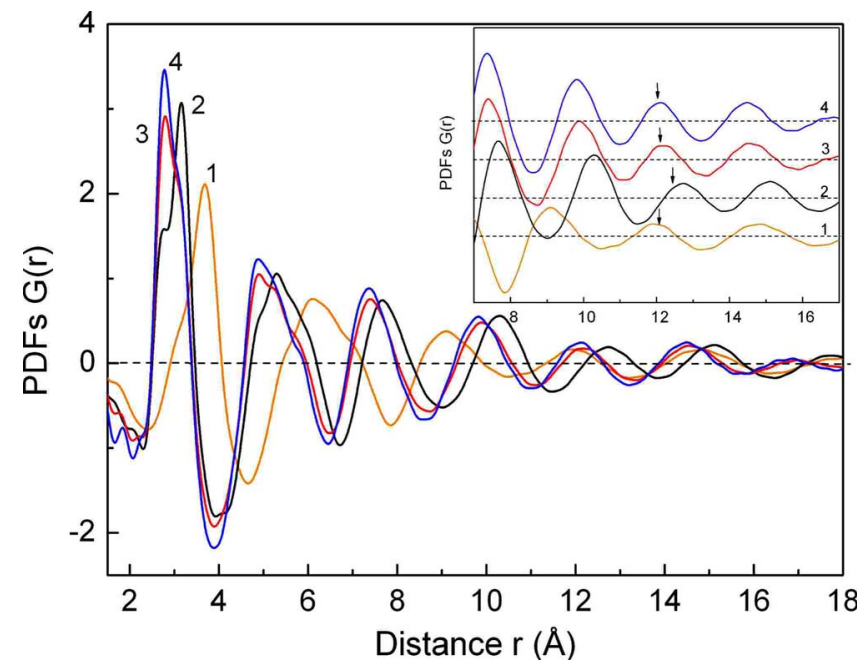

FIG. 3. (Color online) Pair distribution function of total $G(r)$ curves for four BMGs: (1) $\mathrm{La}_{62} \mathrm{Al}_{14}\left(\mathrm{Cu}_{5 / 6} \mathrm{Ag}_{1 / 6}\right){ }_{14} \mathrm{Co}_{5} \mathrm{Ni}_{5}$, (2) $\mathrm{Zr}_{62} \mathrm{Al}_{8} \mathrm{Ni}_{13} \mathrm{Cu}_{17}$, (3) $\mathrm{Zr}_{48} \mathrm{Cu}_{43} \mathrm{Al}_{7} \mathrm{Ag}_{2}$, and (4) $\mathrm{Cu}_{46} \mathrm{Zr}_{46} \mathrm{Al}_{8}$. Inset is the local magnification of $G(r)$ curves, showing the peak area at 11-12 $\AA$ is smaller than others at its both sides.

narily high at the first maximum position $(\sim 2.75 \AA)$, corresponding to the length of $\mathrm{Zr}-\mathrm{Ni}$ and $\mathrm{Zr}-\mathrm{Cu}$ pairs. The elastic moduli evaluated from the position shifts of second to fifth maxima are in the range of 109-74 GPa, showing relatively large scattering. Next, the local strain is calculated from the shift in the average $r$ value $R_{\text {av }}$ for each shell. The $R_{\text {av }}$ stands for an average interatomic distance of one shell from a center atom in $g(r)$ curve, which can be estimated by $R_{\mathrm{av}}$ $=\sum g\left(r_{i}\right) r_{i} / \sum g\left(r_{i}\right)$ and $g(r)=1+G(r) / 4 \pi r \rho_{0}$, where $g(r)$ is the reduced pair correlation function and $\rho_{0}$ is the atomic number density. The relationship between the stress and the strain calculated by this way is shown in Fig. 2(b). Although the abnormally high elastic modulus disappears at the first shell, the stress-strain curves still show very large scattering. Finally, we used the position of $r$ values for $G(r)=0$ to determine the shift in $G(r)$ under tension. There are two $r$ values with $G(r)=0$ at the left and right side of each shell, noted as $1 \mathrm{~L}$ and $1 \mathrm{R}$ for the first shell, $2 \mathrm{~L}$ and $2 \mathrm{R}$ for the second shell, and so on in Fig. 2(c). It is clear that the strains obtained by this way are nearly proportional to the stress and the modulus values deduced from various shells show relatively small fluctuations, to be averagely close to $83 \mathrm{GPa}$ obtained from the $I(q)$ data in Fig. 1. On the basis of above consideration, we conclude that the third way is the best one to deduce local atomic strain from $G(r)$, which was also used in Ref. 11.

Figure 3 shows the total $G(r)$, which are calculated by Fourier transformation of total $S(q)$ data integrated from the whole image plate, for four alloys under zero stress It is found that peak positions in $G(r)$ shift to high $r$ values with increasing atomic size of major component $(\mathrm{Cu} 1.28 \AA$ $<\operatorname{Zr} 1.6 \AA<\operatorname{La} 1.87 \AA)$. Accordingly, the atomic number density, i.e., the number of atoms per unit volume, reduces in the order of $0.06 \AA^{-3}$ for $\mathrm{Cu}_{46} \mathrm{Zr}_{46} \mathrm{Al}_{8}, 0.059 \AA^{-3}$ for $\mathrm{Zr}_{48} \mathrm{Cu}_{43} \mathrm{Al}_{7} \mathrm{Ag}_{2}, \quad 0.053 \AA^{-3}$ for $\mathrm{Zr}_{62} \mathrm{Al}_{8} \mathrm{Ni}_{13} \mathrm{Cu}_{17}$, and $0.038 \AA^{-3}$ for $\mathrm{La}_{62} \mathrm{Al}_{14}\left(\mathrm{Cu}_{5 / 6} \mathrm{Ag}_{1 / 6}\right){ }_{14} \mathrm{Co}_{5} \mathrm{Ni}_{5}$, well corresponding to the trend of decrease in elastic moduli (102 $>91>83>34 \mathrm{GPa}$ ) for these four BMGs. These results suggest that the denser the atomic packing, the higher stiffness is for BMGs studied here. The local atomic strain in the 

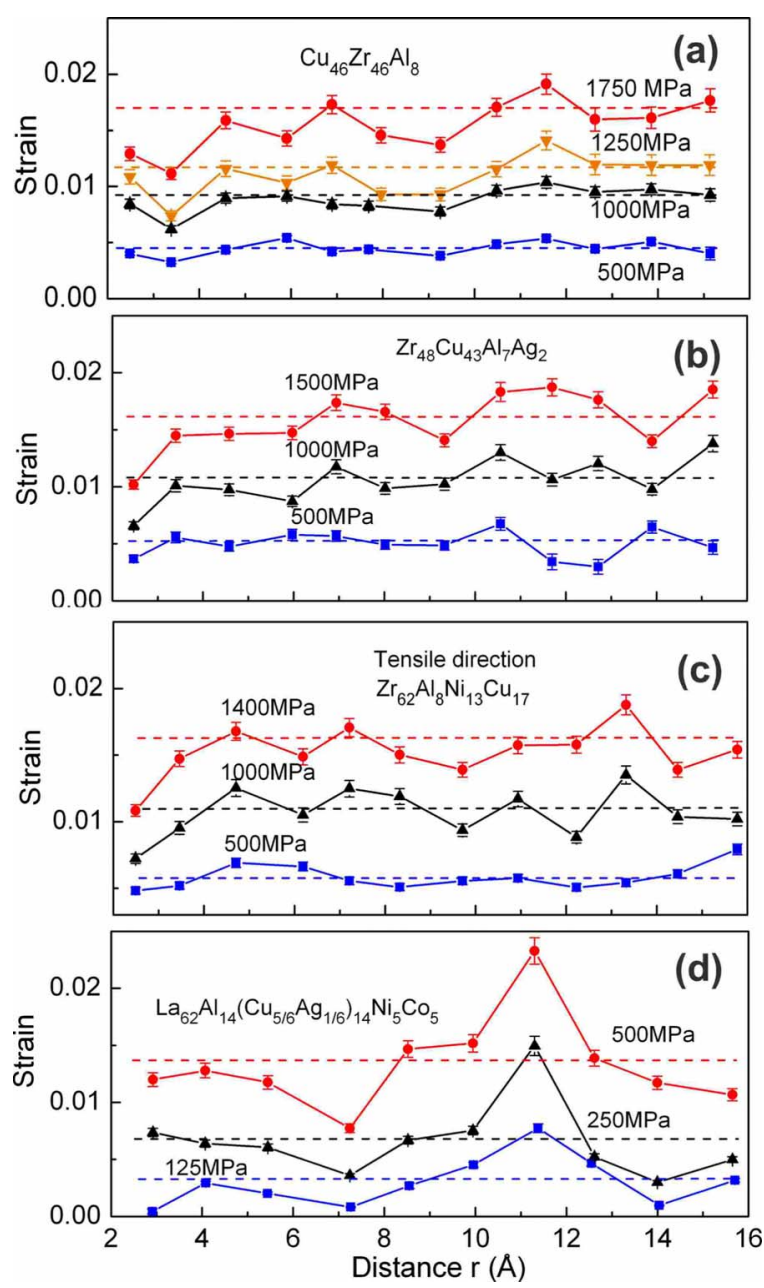

FIG. 4. (Color online) Local strain distribution got from $G(r)$ of tensile direction under different stress for four BMGs. Short dashed lines show the values of microscopic strain under each stress from the $I(q)$ data, as illustrated in Fig. 1.

tensile direction was examined for four BMGs with increasing tensile stress, as illustrated in Figs. 4(a)-4(d). It shows that the local strain of four BMGs under each stress is roughly consistent with the microscopic strain (shown by short dashed line) obtained from $I(q)$ data. In addition, the local atomic strain increases in a homogeneous way when the stress is low, like the case below $500 \mathrm{MPa}$ for Zr-based and CuZr-based BMGs. The fluctuation in local atomic strain becomes more distinct when the stress increases, which mainly appears in the two ranges of about $4-8$ and 10-14 $\AA$. For $\mathrm{La}_{62} \mathrm{Al}_{14}\left(\mathrm{Cu}_{5 / 6} \mathrm{Ag}_{1 / 6}\right)_{14} \mathrm{Co}_{5} \mathrm{Ni}_{5} \mathrm{BMG}$, the largest oscillation in local atomic strain along the tensile direction is concentrated at the position around $r=11 \AA$ even at low stress. Similar results were also reported for compression of BMGs, in which the local strain at $r$ range of below $4 \AA$ is smaller than the strain at $r$ range of $4-10 \AA$ of a $\mathrm{Mg}-\mathrm{Cu}-\mathrm{Y} \mathrm{BMG}^{10}$

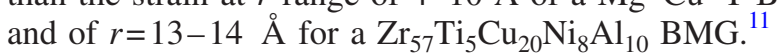

It is commonly believed that the structure of amorphous alloys can be described by randomly packed clusters, ${ }^{15,16}$ which could be tricapped trigonal prisms, icosahedron, Frank-Kasper-type polyhedra, etc. No translational symmetry exists for clusters packing in BMGs. However, several clusters packing together could form a supercluster or medium range order. These superclusters connect each other randomly to fill the three dimensional space while excess open volume exists between superclusters. Thus, we pro- posed that the local atomic strain fluctuations in the ranges of 4-8 and 10-14 $\AA$ observed in Fig. 4 could be linked with different responses from clusters and superclusters in BMGs under high tensile stresses, respectively. The large oscillation at $11 \AA$ in La-based BMG could indicate the high open volume existing between the superclusters. This scenario mentioned above is supported by the fact that the peak areas of $G(r)$ in the range of about 10-13 $\AA$ for four BMGs studied are indeed smaller than neighbors at both sides as shown by inset of Fig. 3. Furthermore, a recent study on the distribution of free volume in a $\mathrm{Zr}-\mathrm{Ni} \mathrm{MG}$ also supported this scenario. ${ }^{17}$

In summary, the tensile behavior of $\mathrm{Zr}_{62} \mathrm{Al}_{8} \mathrm{Ni}_{13} \mathrm{Cu}_{17}$, $\mathrm{Cu}_{46} \mathrm{Zr}_{46} \mathrm{Al}_{8}, \mathrm{Zr}_{48} \mathrm{Cu}_{43} \mathrm{Al}_{7} \mathrm{Ag}_{2}$, and $\mathrm{La}_{62} \mathrm{Al}_{14}\left(\mathrm{Cu}_{5 / 6} \mathrm{Ag}_{1 / 6}\right)_{14}$ $\times \mathrm{Co}_{5} \mathrm{Ni}_{5}$ BMGs has been investigated by using in situ synchrotron radiation diffraction technique. It is found that the heterogeneity in local strain appears in these four BMGs with increasing tensile stress. The largest fluctuation happens in the range of 10-14 $\AA$ that seems to be the boundary between superclusters, where excess open volume may accumulate. This suggests that relatively homogeneous atomic packing within the medium range order may promote plasticity of monolithic BMGs.

The authors would like to thank the HASYLAB staff at Hamburg, Germany, for the assistance during the measurements at BW5. Financial support from the National Natural Science Foundation of China (Grant Nos. 50425102, 50601021, 50701038, 60776014, 60876002, and 10804096), Zhejiang University-Helmholtz cooperation fund, the Ministry of Education of China (Program for Changjiang Scholars), the Department of Science and Technology of Zhejiang province, and Zhejiang University are gratefully acknowledged.

${ }^{1}$ C. C. Hays, C. P. Kim, and W. L. Johnson, Phys. Rev. Lett. 84, 2901 (2000).

${ }^{2}$ J. Schroers and W. L. Johnson, Phys. Rev. Lett. 93, 255506 (2004).

${ }^{3}$ C. A. Schuh, T. C. Hufnagel, and U. Ramamurty, Acta Mater. 55, 4067 (2007).

${ }^{4}$ X. D. Wang, L. Yang, J. Z. Jiang, K. Saksl, H. Franz, H.-J. Fecht, Y. G. Liu, and H. S. Xian, J. Mater. Res. 22, 2454 (2007); Y. H. Liu, G. Wang, R. J. Wang, D. Q. Zhao, M. X. Pan, and W. H. Wang, Science 315, 1385 (2007).

${ }^{5}$ R. D. Conner, W. L. Johnson, N. E. Paton, and W. D. Nix, J. Appl. Phys. 94, 904 (2003).

${ }^{6}$ D. C. Hofmann, J. Y. Suh, A. Wiest, G. Duan, M. L. Lind, M. D. Demetriou, and W. L. Johnson, Nature (London) 451, 1085 (2008).

${ }^{7}$ A. S. Argon, Acta Metall. 27, 47 (1979).

${ }^{8}$ F. Spaepen, Acta Metall. 25, 407 (1977).

${ }^{9}$ S. C. Lee, C. M. Lee, J. W. Yang, and J. C. Lee, Scr. Mater. 58, 591 (2008).

${ }^{10}$ H. F. Poulsen, J. A. Wert, J. Neuefeind, V. Honkimaki, and M. Daymond, Nature Mater. 4, 33 (2005).

${ }^{11}$ T. C. Hufnagel, R. T. Ott, and J. Almer, Phys. Rev. B 73, 064204 (2006)

${ }^{12}$ A. R. Yavari, A. L. Moulec, A. Inoue, N. Nishiyama, N. Lupu, E. Matsubara, W. J. Botta, G. Vaughan, M. D. Michiel, and A. Kvick, Acta Mater. 53, 1611 (2005)

${ }^{13}$ R. T. Ott, M. J. Kramer, M. F. Besser, and D. J. Sordelet, Acta Mater. 54, 2463 (2006).

${ }^{14}$ X. D. Wang, J. Bednarcik, K. Saksl, H. Franz, Q. P. Cao, and J. Z. Jiang, Appl. Phys. Lett. 91, 081913 (2007).

${ }^{15}$ D. B. Miracle, Nature Mater. 3, 697 (2004).

${ }^{16}$ H. W. Sheng, W. K. Luo, F. M. Alamgir, J. M. Bai, and E. Ma, Nature (London) 439, 419 (2006).

${ }^{17}$ X. J. Liu, G. L. Chen, X. Hui, T. Liu, and Z. P. Lu, Appl. Phys. Lett. 93 011911 (2008). 